\title{
Rayleigh Waves in a Rotating Orthotropic Micropolar Elastic Solid Half-Space
}

\author{
Baljeet Singh, ${ }^{1}$ Ritu Sindhu, ${ }^{2}$ and Jagdish Singh ${ }^{2}$ \\ ${ }^{1}$ Department of Mathematics, Post Graduate Government College, Sector 11, Chandigarh 160 011, India \\ ${ }^{2}$ Department of Mathematics, Maharshi Dayanand University, Rohtak, 124001 Haryana, India \\ Correspondence should be addressed to Baljeet Singh; bsinghgcl1@gmail.com
}

Received 12 March 2013; Revised 7 May 2013; Accepted 13 May 2013

Academic Editor: Rudolf A. Treumann

Copyright (c) 2013 Baljeet Singh et al. This is an open access article distributed under the Creative Commons Attribution License, which permits unrestricted use, distribution, and reproduction in any medium, provided the original work is properly cited.

\begin{abstract}
A problem on Rayleigh wave in a rotating half-space of an orthotropic micropolar material is considered. The governing equations are solved for surface wave solutions in the half space of the material. These solutions satisfy the boundary conditions at free surface of the half-space to obtain the frequency equation of the Rayleigh wave. For numerical purpose, the frequency equation is approximated. The nondimensional speed of Rayleigh wave is computed and shown graphically versus nondimensional frequency and rotation-frequency ratio for both orthotropic micropolar elastic and isotropic micropolar elastic cases. The numerical results show the effects of rotation, orthotropy, and nondimensional frequency on the nondimensional speed of the Rayleigh wave.
\end{abstract}

\section{Introduction}

Material response to external stimuli depends heavily on the motions of its inner structures. Classical elasticity does not include this effect, where only translation degrees of freedom of material point of body are considered. Eringen [1] developed the linear micropolar theory of elasticity, which included the intrinsic rotations of the microstructure. It provides a model which can support body and surface couples and display high frequency optical branch of the wave spectrum. For engineering applications, it can model composites with rigid chopped fibres, elastic solid with rigid granular inclusions, and other industrial materials such as liquid crystals.

The assumptions of isotropy in a solid medium may not capture some of significant features of the continuum responses of soils, geological materials, and composites. Iesan [2-4] studied some static problems in orthotropic micropolar elasticity. Kumar and Choudhary $[5,6]$ studied the mechanical sources and dynamic behaviour of orthotropic micropolar elastic medium. Kumar and Chaudhary [7] studied the plane strain problem in a homogeneous orthotropic micropolar elastic solid. Kumar and Ailawalia [8] studied the response of a micropolar cubic crystal due to various sources. Kumar and
Gupta [9] studied the propagation of waves in transversely isotropic micropolar generalized thermoelastic half-space. Singh [10] investigated the two-dimensional plane wave propagation in an orthotropic micropolar elastic solid.

Surface waves in elastic solids were first studied by Rayleigh [11] for an isotropic elastic solid. The extension of surface wave analysis and other wave propagation problems to anisotropic elastic materials has been the subject of many studies; see, for example, [12-21]. The aim of the present paper is to study the propagation of Rayleigh wave in a rotating orthotropic micropolar elastic solid half space. The frequency equation of the Rayleigh wave is obtained. The speed of Rayleigh wave is computed with the help of approximated frequency equation. The effects of orthotropy, non-dimensional frequency, and rotation are shown graphically on the nondimensional speed of the Rayleigh wave.

\section{Formulation of the Problem and Solution}

We consider a homogeneous and orthotropic medium of an infinite extent with Cartesian coordinate system $(x, y, z)$. We restrict our study to the plane strain parallel to $x y$-plane, with the displacement vector $\mathbf{u}=\left(u_{1}, u_{2}, 0\right)$ and microrotation 
vector $\phi=\left(0,0, \phi_{3}\right)$. Following Eringen [22] and Schoenberg and Censor [23], the field equations in $x y$-plane for homogeneous and rotating orthotropic micropolar solid in absence of body forces and couples are written as

$$
\begin{gathered}
A_{11} u_{1,11}+\left(A_{12}+A_{78}\right) u_{2,12}+A_{88} u_{1,22}-K_{1} \phi_{3,2} \\
=\rho\left[\frac{\partial^{2} u_{1}}{\partial t^{2}}-\Omega^{2} u_{1}-2 \Omega \frac{\partial u_{2}}{\partial t}\right], \\
\left(A_{12}+A_{78}\right) u_{1,12}+A_{77} u_{2,11}+A_{22} u_{2,22}-K_{2} \phi_{3,1} \\
=\rho\left[\frac{\partial^{2} u_{2}}{\partial t^{2}}-\Omega^{2} u_{2}+2 \Omega \frac{\partial u_{1}}{\partial t}\right], \\
B_{66} \phi_{3,11}+B_{44} \phi_{3,22}-\chi \phi_{3}+K_{1} u_{1,2}+K_{2} u_{2,1}=\rho j \ddot{\phi}_{3},
\end{gathered}
$$

where

$$
K_{1}=A_{78}-A_{88}, \quad K_{2}=A_{77}-A_{78}, \quad \chi=K_{2}-K_{1} .
$$

We consider the following surface wave solutions

$$
\left\{u_{1}, u_{2}, \phi_{3}\right\}=\left\{\bar{u}_{1}(y), \bar{u}_{2}(y), \bar{\phi}_{3}(y)\right\} e^{i k(x-c t)},
$$

where $k$ is the wave number, $c$ is phase velocity of the wave, and $\omega=k c$ is the angular frequency. Making use of (5) in (1) to (3), we obtain three homogeneous equations in $\bar{u}_{1}(y)$, $\bar{u}_{2}(y)$, and $\bar{\phi}_{3}(y)$, which have nontrivial solutions if

$$
\alpha D^{6}-\beta D^{4}+\gamma D^{2}-\delta=0,
$$

where $D=d / d y$ and $\alpha, \beta, \gamma$, and $\delta$ are given in Appendix.

Let $m_{1}, m_{2}$, and $m_{3}$ be the roots of auxiliary equation (6). Then, the general solutions of (6) are written as

$$
\begin{aligned}
u_{1}= & \left(A_{1} e^{-m_{1} y}+A_{2} e^{-m_{2} y}+A_{3} e^{-m_{3} y}+A_{4} e^{m_{1} y}\right. \\
& \left.+A_{5} e^{m_{2} y}+A_{6} e^{m_{3} y}\right) e^{i k(x-c t)} \\
u_{2}= & \zeta_{1} A_{1} e^{-m_{1} y}+\zeta_{2} A_{2} e^{-m_{2} y}+\zeta_{3} A_{3} e^{-m_{3} y}+\zeta_{1} A_{4} e^{m_{1} y} \\
& \left.+\zeta_{2} A_{5} e^{m_{2} y}+\zeta_{3} A_{6} e^{m_{3} y}\right) e^{i k(x-c t)}, \\
\phi_{3}= & \eta_{1} A_{1} e^{-m_{1} y}+\eta_{2} A_{2} e^{-m_{2} y}+\eta_{3} A_{3} e^{-m_{3} y}+\eta_{1} A_{4} e^{m_{1} y} \\
& \left.+\eta_{2} A_{5} e^{m_{2} y}+\eta_{3} A_{6} e^{m_{3} y}\right) e^{i k(x-c t)},
\end{aligned}
$$

where

$$
\begin{gathered}
m_{1}^{2}+m_{2}^{2}+m_{3}^{2}=\frac{\beta}{\alpha} \\
m_{1}^{2} m_{2}^{2}+m_{2}^{2} m_{3}^{2}+m_{3}^{2} m_{1}^{2}=\frac{\gamma}{\alpha}, \\
m_{1}^{2} m_{2}^{2} m_{3}^{2}=\frac{\delta}{\alpha}
\end{gathered}
$$

and the expressions for $\zeta_{1}, \zeta_{2}$, and $\zeta_{3}$ and $\eta_{1}, \eta_{2}$, and $\eta_{3}$ are given in the Appendix.
With the use of the radiation conditions $u_{1} \rightarrow 0, u_{2} \rightarrow$ $0, \varphi_{3} \rightarrow 0$ as $y \rightarrow \infty$, we obtain the particular solutions for medium $(y>0)$ as

$$
\begin{gathered}
u_{1}=\left(A_{1} e^{-m_{1} y}+A_{2} e^{-m_{2} y}+A_{3} e^{-m_{3} y}\right) e^{i k(x-c t)}, \\
u_{2}=\left(\zeta_{1} A_{1} e^{-m_{1} y}+\zeta_{2} A_{2} e^{-m_{2} y}+\zeta_{3} A_{3} e^{-m_{3} y}\right) e^{i k(x-c t)}, \\
\phi_{3}=\left(\eta_{1} A_{1} e^{-m_{1} y}+\eta_{2} A_{2} e^{-m_{2} y}+\eta_{3} A_{3} e^{-m_{3} y}\right) e^{i k(x-c t)} .
\end{gathered}
$$

\section{Boundary Conditions}

The mechanical boundary conditions at $y=0$ are the vanishing of normal force stress tangential force stress; and tangential couple stress that is,

$$
t_{22}=0, \quad t_{21}=0, \quad m_{23}=0
$$

where

$$
\begin{gathered}
t_{22}=A_{12} \frac{\partial u_{1}}{\partial x}+A_{22} \frac{\partial u_{2}}{\partial y} \\
t_{21}=A_{78} \frac{\partial u_{2}}{\partial x}+A_{88} \frac{\partial u_{1}}{\partial y}+\left(A_{88}-A_{78}\right) \phi_{3}, \\
m_{23}=B_{44} \frac{\partial \phi_{3}}{\partial y}
\end{gathered}
$$

The solutions given by (11) to (13) satisfy the boundary conditions (14) at $y=0$, and we obtain the following frequency equation:

$$
\begin{aligned}
\frac{A_{12}}{A_{22}} & \frac{A_{78}}{A_{88}} \sum \frac{m_{1}}{k} \frac{\eta_{1}}{k}\left(\zeta_{2}-\zeta_{3}\right)+i \frac{A_{12}}{A_{22}} \sum \frac{m_{1}}{k} \frac{m_{2}}{k}\left(\frac{\eta_{1}}{k}-\frac{\eta_{2}}{k}\right) \\
& +i \frac{A_{12}}{A_{22}} \frac{K_{1}}{A_{88}} \sum \frac{m_{1}}{k} \frac{\eta_{1}}{k}\left(\frac{\eta_{2}}{k}-\frac{\eta_{3}}{k}\right) \\
& +i \frac{A_{78}}{A_{88}} \sum \frac{m_{1}}{k} \frac{m_{2}}{k} \zeta_{3}\left(\frac{\eta_{2}}{k} \zeta_{1}-\frac{\eta_{1}}{k} \zeta_{2}\right) \\
& +\frac{K_{1}}{A_{88}} \sum \frac{m_{1}}{k} \frac{m_{2}}{k} \frac{\eta_{3}}{k}\left(\frac{\eta_{1}}{k} \zeta_{2}-\frac{\eta_{2}}{k} \zeta_{1}\right) \\
& +\sum \frac{m_{1}}{k} \frac{m_{2}}{k} \frac{m_{3}}{k}\left(\frac{\eta_{1}}{k} \zeta_{2}-\frac{\eta_{2}}{k} \zeta_{1}\right)=0 .
\end{aligned}
$$

\section{Particular Case}

The frequency equation (16) reduces to the frequency equation for an isotropic rotating micropolar elastic case, if we take

$$
\begin{gathered}
A_{11}=A_{22}=\lambda+2 \mu+\kappa, \\
A_{77}=A_{88}=\mu+\kappa, \quad A_{12}=\lambda, \quad A_{78}=\mu, \\
B_{44}=B_{66}=\gamma, \quad-K_{1}=K_{2}=\frac{\chi}{2}=\kappa .
\end{gathered}
$$




\section{Numerical Results and Discussion}

From relations (8) to (10), we obtain the following approximated roots:

$$
\begin{gathered}
\frac{m_{1}^{2}}{k^{2}} \cong \frac{\left(A_{11}-\rho c^{2} \Omega^{*}\right)}{A_{88}}, \\
\frac{m_{2}^{2}}{k^{2}} \cong \frac{\left(A_{77}-\rho c^{2} \Omega^{*}\right)}{A_{22}}, \\
\frac{m_{3}^{2}}{k^{2}} \cong \frac{\left(B_{66}+\rho j c^{2}+\left(\chi / k^{2}\right)\right)}{B_{44}} .
\end{gathered}
$$

With the help of (18) to (20), the frequency equation (16) reduces to the approximated frequency equation for an orthotropic rotating micropolar elastic case. The approximated frequency equation is used to compute the non-dimensional speed of the Rayleigh wave in orthotropic micropolar solid half-space for the following arbitrary physical constants:

$$
\begin{gathered}
A_{11}=11.65 \times 10^{10} \mathrm{Nm}^{-2}, \\
A_{22}=11.71 \times 10^{10} \mathrm{Nm}^{-2}, \\
A_{12}=7.69 \times 10^{10} \mathrm{Nm}^{-2}, \\
A_{77}=1.99 \times 10^{10} \mathrm{Nm}^{-2}, \\
A_{78}=1.98 \times 10^{10} \mathrm{Nm}^{-2}, \\
A_{88}=2.01 \times 10^{10} \mathrm{Nm}^{-2}, \\
B_{44}=0.036 \times 10^{10} \mathrm{~N}, \quad B_{66}=0.037 \times 10^{10} \mathrm{~N}, \\
\rho=2.19 \times 10^{3} \mathrm{Kg} \mathrm{m}^{-3}, \quad j=0.000196 \mathrm{~m}^{2} .
\end{gathered}
$$

The non-dimensional speed of the Rayleigh wave is also computed for isotropic micropolar elastic case with following relevant parameters [24]:

$$
\begin{gathered}
\lambda=7.59 \times 10^{10} \mathrm{Nm}^{-2}, \quad \mu=1.89 \times 10^{10} \mathrm{Nm}^{-2}, \\
\kappa=0.0149 \times 10^{10} \mathrm{Nm}^{-2}, \quad \gamma=0.0268 \times 10^{9} \mathrm{~N}, \\
\rho=2.19 \times 10^{3} \mathrm{Kg} \mathrm{m}^{-3}, \quad j=0.000196 \mathrm{~m}^{2} .
\end{gathered}
$$

The non-dimensional speed $c^{*}\left(=\rho c^{2} / A_{22}\right)$ of Rayleigh wave is computed for orthotropic micropolar elastic case and isotropic micropolar elastic case for different values of nondimensional frequency $\omega^{*}\left(=\omega^{2} /[\chi / \rho j]\right)$ and rotationfrequency ratio $\Omega / \omega$.

The non-dimensional speed $c^{*}$ is plotted against the rotation-frequency ratio $\Omega / \omega$, when non-dimensional frequency $\omega^{*}=5,10$, and 20 . The speed $c^{*}$ decreases with the increase in value of rotation-frequency ratio $\Omega / \omega$. For each value of rotation-frequency ratio $\Omega / \omega$, the speed $c^{*}$ increases with the increase in value of non-dimensional frequency $\omega^{*}$. The effect of non-dimensional frequency $\omega^{*}$ on non-dimensional speed $c^{*}$ decreases with the increase in values of $\Omega / \omega$. The variations

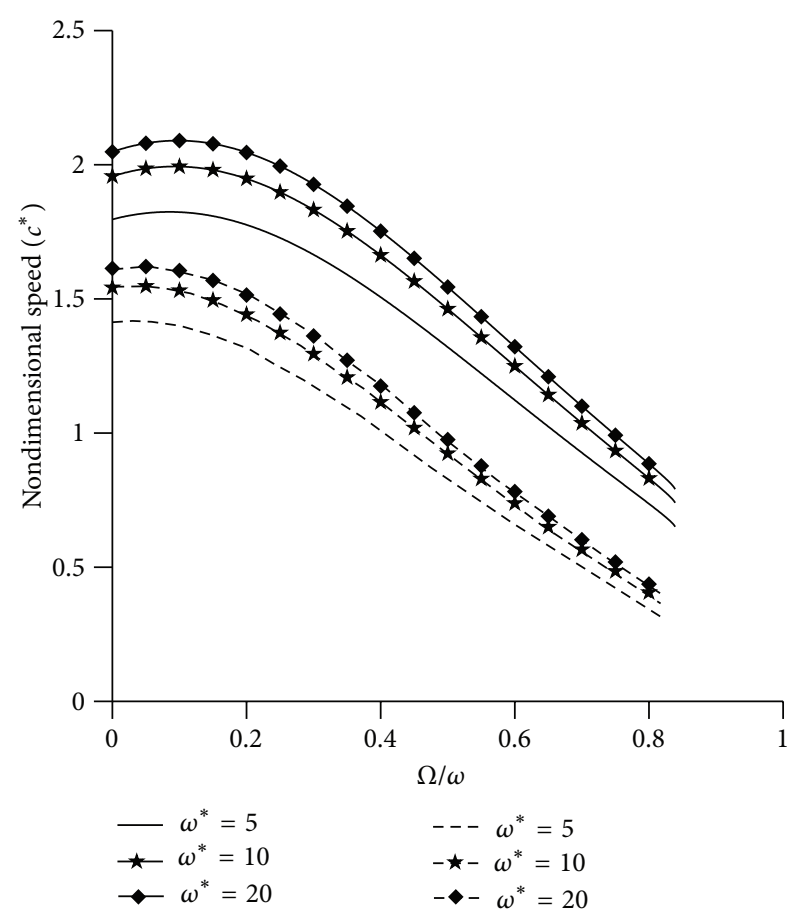

FIGURE 1: Variations of the nondimensional speed of Rayleigh wave against rotation-frequency ratio (solid lines:orthotropic micropolar elastic case, dotted lines:isotropic micropolar elastic case).

showing the effect of orthotropy on non-dimensional speed of Rayleigh wave are shown in Figure 1, where solid lines and dotted lines correspond to the orthotropic micropolar elastic case and isotropic micropolar elastic case, respectively.

The speed $c^{*}$ is also plotted against the non-dimensional frequency $\omega^{*}$, when rotation-frequency ratio $\Omega / \omega=0.2,0.4$, and 0.6 . The speed $c^{*}$ of Rayleigh wave increases sharply with the increase in value of non-dimensional frequency $\omega^{*}$ for both orthotropic micropolar elastic case and isotropic micropolar elastic case. Beyond $\omega^{*}=4$, it increases slowly in the both cases. Here, the speed $c^{*}$ decreases with the increase in values of $\Omega / \omega$ in each case. The effect of rotation on nondimensional speed $c^{*}$ increases with the increase in value of non-dimensional frequency $\omega^{*}$. The variations showing the effect of orthotropy on non-dimensional speed of Rayleigh wave are shown in Figure 2, where solid lines and dotted lines correspond to the orthotropic micropolar elastic case and isotropic micropolar elastic case, respectively.

\section{Conclusion}

The propagation of Rayleigh wave is studied in an orthotropic micropolar elastic solid half-space, where we obtained the required approximated frequency equation of Rayleigh wave. The non-dimensional speed $c^{*}\left(=\rho c^{2} / A_{22}\right)$ is computed for certain ranges of non-dimensional frequency $\omega^{*}$ and rotation-frequency ratio $\Omega / \omega$. The comparison of solid and dotted line curves in the figures reveals the effect of orthotropy, 


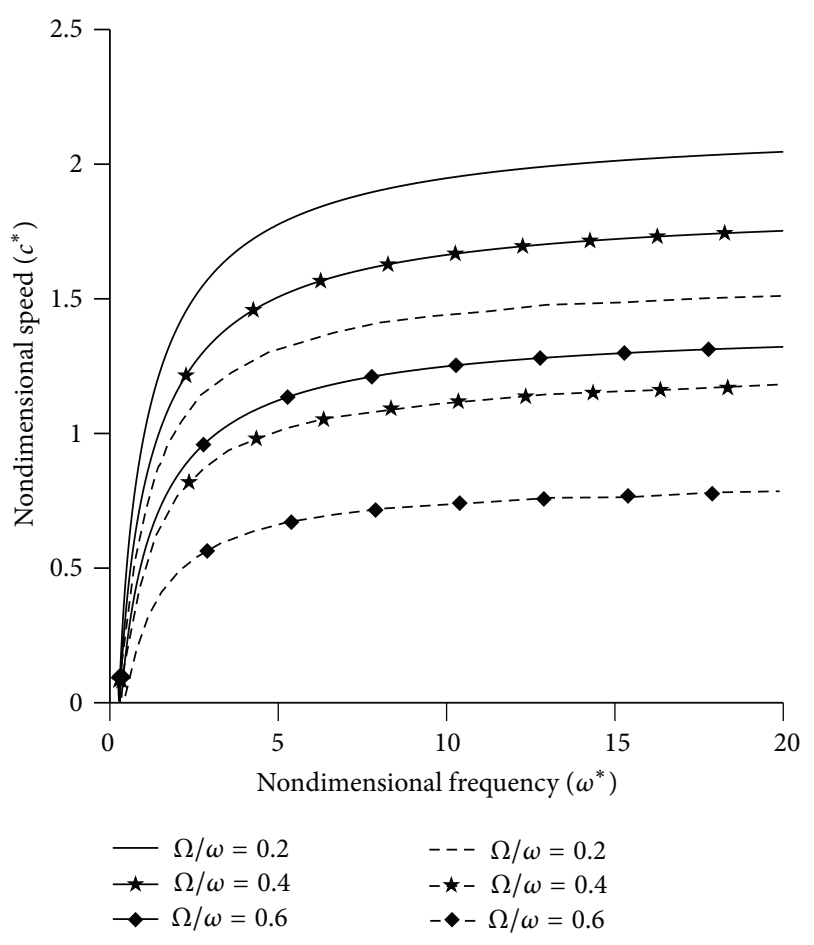

FIGURE 2: Variations of the non-dimensional speed of Rayleigh wave against non-dimensional frequency $\omega^{*}$ (solid lines:orthotropic micropolar elastic case, dotted lines:isotropic micropolar elastic case).

rotation, and non-dimensional frequency $\omega^{*}$ on the nondimensional speed $c^{*}$ of the Rayleigh wave in an orthotropic micropolar elastic solid half-space.

\section{Appendix}

The values of $\alpha, \beta, \gamma$, and $\delta$ are given as

$$
\begin{gathered}
\alpha=A_{22} A_{88} B_{44}, \\
\beta=k^{2}\left(A_{22} A_{88} L_{4}+A_{88} B_{44} L_{3}\right. \\
\left.+A_{22} B_{44} L_{1}-B_{44} L_{2}^{2}\right)-A_{22} K_{1}^{2}, \\
\gamma=k^{4}\left(-L_{2}^{2} L_{4}+L_{3} L_{4} A_{88}+L_{1} L_{4} A_{22}\right. \\
\left.+L_{1} L_{3} B_{44}-4\left(\rho c^{2}\right)^{2}\left(\frac{\Omega}{\omega}\right)^{2} B_{44}\right) \\
+k^{2}\left(-K_{2}^{2} A_{88}+2 K_{1} K_{2} L_{2}-K_{1}^{2} L_{3}\right), \\
k^{6}\left[L_{1} L_{3} L_{4}-4\left(\rho c^{2}\right)^{2}\left(\frac{\Omega^{2}}{\omega}\right)^{2} L_{4}\right]-k^{4} L_{1} K_{2}^{2}, \\
L_{1} A_{11}-\rho c^{2} \Omega^{*}, \quad L_{2}=A_{12}+A_{78}, \\
L_{3}=A_{77}-\rho c^{2} \Omega^{*}, \\
L_{4}=B_{66}+\rho j c^{2}+\frac{\chi}{k^{2}}, \quad \Omega^{*}=1+\frac{\Omega^{2}}{\omega^{2}} .
\end{gathered}
$$

The values of $\zeta_{1}, \zeta_{2}$, and $\zeta_{3}$ and $\eta_{1}, \eta_{2}$, and $\eta_{3}$ are obtained as

$$
\begin{aligned}
& \zeta_{i}=i\left[\left(\frac{m_{i}}{k}\left(\frac{m_{i}}{k} \frac{A_{12}+A_{78}}{A_{88}}-\frac{2 \rho c^{2}}{A_{88}} \frac{\Omega}{\omega}\right)\right.\right. \\
& \left.-\frac{K_{2}}{K_{1}}\left(\frac{m_{i}^{2}}{k^{2}}-\frac{A_{11}-\rho c^{2} \Omega^{*}}{A_{88}}\right)\right) \\
& \times\left(\frac{K_{2}}{K_{1}}\left(\frac{m_{i}}{k} \frac{A_{12}+A_{78}}{A_{88}}+\frac{2 \rho c^{2}}{A_{88}} \frac{\Omega}{\omega}\right)\right. \\
& \left.\left.+\frac{m_{i}}{k} \frac{A_{22}}{A_{88}}\left(\frac{m_{i}^{2}}{k^{2}}-\frac{A_{77}-\rho c^{2} \Omega^{*}}{A_{22}}\right)\right)^{-1}\right], \\
& \frac{\eta_{i}}{k} \\
& =\left(\left[\left(\frac{m_{i}^{2}}{k^{2}} \frac{\left(A_{12}+A_{78}\right)^{2}}{A_{22} A_{88}}-4 \frac{\left(\rho c^{2}\right)^{2}}{A_{22} A_{88}}\left(\frac{\Omega}{\omega}\right)^{2}\right)\right.\right. \\
& \left.\left.-\left(\frac{m_{i}^{2}}{k^{2}}-\frac{A_{11}-\rho c^{2} \Omega^{*}}{A_{88}}\right)\left(\frac{m_{i}^{2}}{k^{2}}-\frac{A_{77}-\rho c^{2} \Omega^{*}}{A_{22}}\right)\right]\right) \\
& \times\left(\left[\frac{K_{2}}{A_{88}}\left(\frac{m_{i}}{k} \frac{A_{12}+A_{78}}{A_{22}}+2 \frac{\rho c^{2}}{A_{22}} \frac{\Omega}{\omega}\right)\right.\right. \\
& \left.\left.+\frac{m_{i}}{k} \frac{K_{1}}{A_{88}}\left(\frac{m_{i}^{2}}{k^{2}}-\frac{A_{77}-\rho c^{2} \Omega^{*}}{A_{22}}\right)\right]\right)^{-1} .
\end{aligned}
$$

\section{References}

[1] A. C. Eringen, "Linear theory of micropolar elasticity," Journal of Mathematics and Mechanics, vol. 15, pp. 909-924, 1966.

[2] D. Iesan, "The plane micropolar strain of orthotropic elastic solids," Archives of Mechanics, vol. 25, no. 3, pp. 547-561, 1973.

[3] D. Iesan, "Torsion of anisotropic elastic cylinders," Zeitschrift für Angewandte Mathematik und Mechanik, vol. 54, no. 12, pp. 773$779,1974$.

[4] D. Iesan, "Bending of orthotropic micropolar elastic beams by terminal couples," Analele Știinţifice ale Universităţii Al. I. Cuza din Iaşi, vol. 25, pp. 411-418, 1974.

[5] R. Kumar and S. Choudhary, "Mechanical sources in orthotropic micropolar continua," Proceedings of the Indian Academy of Sciences, vol. 111, no. 2, pp. 133-141, 2002.

[6] R. Kumar and S. Choudhary, "Dynamical behaviour of orthotropic micropolar elastic medium," Journal of Vibration and Control, vol. 8, no. 8, pp. 1053-1069, 2002.

[7] R. Kumar and S. Choudhary, "Response of orthotropic micropolar elastic medium due to time harmonic source," Sadhana, vol. 29, pp. 112-116, 2004.

[8] R. Kumar and P. Ailawalia, "Deformation in micropolar cubic crystal due to various sources," International Journal of Solids and Structures, vol. 42, no. 23, pp. 5931-5944, 2005.

[9] R. Kumar and R. R. Gupta, "Propagation of waves in transversely isotropic micropolar generalized thermoelastic half 
space," International Communications in Heat and Mass Transfer, vol. 37, no. 10, pp. 1452-1458, 2010.

[10] B. Singh, "Wave propagation in an orthotropic micropolar elastic solid," International Journal of Solids and Structures, vol. 44, no. 11-12, pp. 3638-3645, 2007.

[11] L. Rayleigh, "On waves propagated along the plane surface of an elastic solid," Proceedings of the London Mathematical Society, vol. 17, pp. 4-11, 1885.

[12] D. L. Anderson, "Elastic wave propagation in layered anisotropic media," Journal of Geophysical Research, vol. 66, pp. 29532963, 1961.

[13] D. M. Barnett and J. Lothe, "Free surface (Rayleigh) waves in anisotropic elastic half-spaces: the surface impedance method," Proceedings of the Royal Society A, vol. 402, pp. 135-152, 1985.

[14] P. Chadwick and G. D. Smith, "Foundations of the theory of surface waves in anisotropic elastic materials," Advances in Applied Mechanics, vol. 17, pp. 303-376, 1977.

[15] M. Destrade, "The explicit secular equation for surface acoustic waves in monoclinic elastic crystals," Journal of the Acoustical Society of America, vol. 109, no. 4, pp. 1398-1402, 2001.

[16] M. A. Dowaikh and R. W. Ogden, "On surface waves and deformations in a compressible elastic half-space," Stability \& Applied Analysis of Continuous Media, vol. 1, pp. 27-45, 1991.

[17] M. J. P. Musgrave, "The propagation of elastic waves in crystals and other anisotropic media," Reports on Progress in Physics, vol. 22, pp. 74-96, 1959.

[18] R. W. Ogden and P. C. Vinh, "On Rayleigh waves in incompressible orthotropic elastic solids," Journal of the Acoustical Society of America, vol. 115, no. 2, pp. 530-533, 2004.

[19] D. Royer and E. Dieulesaint, "Rayleigh wave velocity and displacement in orthorhombic, tetragonal, hexagonal and cubic crystals," Journal of the Acoustical Society of America, vol. 76, no. 5, pp. 1438-1444, 1984.

[20] T. C. T. Ting, "An explicit secular equation for surface waves in an elastic material of general anisotropy," Quarterly Journal of Mechanics and Applied Mathematics, vol. 55, pp. 297-311, 2002.

[21] T. C. T. Ting, "Explicit secular equations for surface waves in monoclinic materials with the symmetry plane at $\mathrm{x}_{1}=0, \mathrm{x}_{2}=0$ or $\mathrm{x}_{3}=0$," Proceedings of the Royal Society A, vol. 458, no. 2021, pp. 1017-1031, 2002.

[22] A. C. Eringen, "Theory of micropolar elasticity," in Fracture, vol. 2, pp. 621-729, Academic Press, New York, NY, USA, 1968.

[23] M. Schoenberg and D. Censor, "Elastic waves in rotating media," Quarterly of Applied Mathematics, vol. 31, no. 1, pp. 115-125, 1973.

[24] R. D. Gauthier, "Experimental investigation on micropolar media," in Mechanics of Micropolar Media, O. Brulin and R. K. T. Hsieh, Eds., World Scientific, Singapore, 1982. 

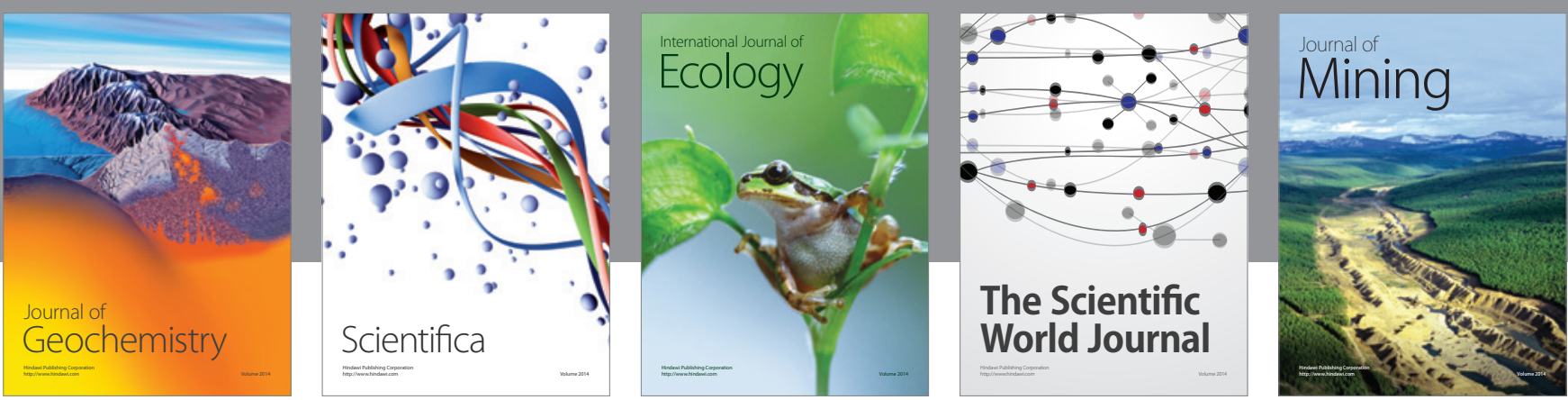

The Scientific World Journal
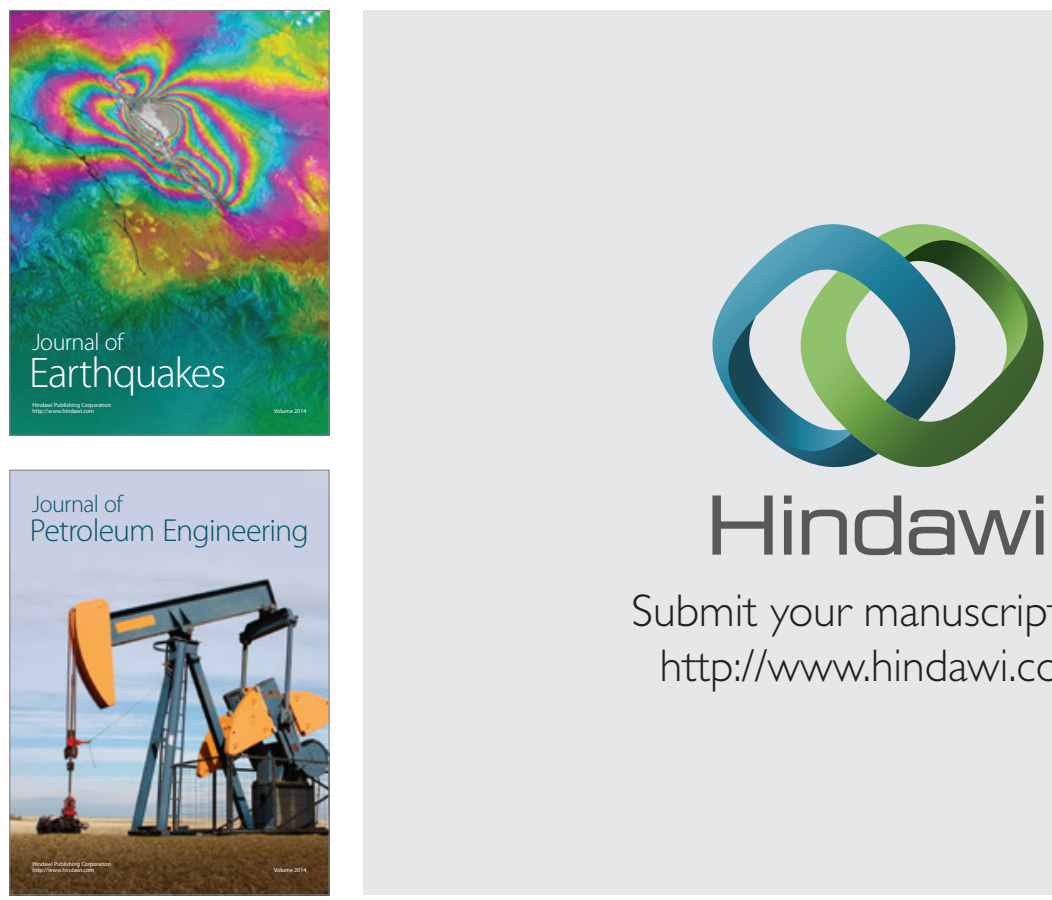

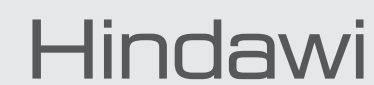

Submit your manuscripts at

http://www.hindawi.com
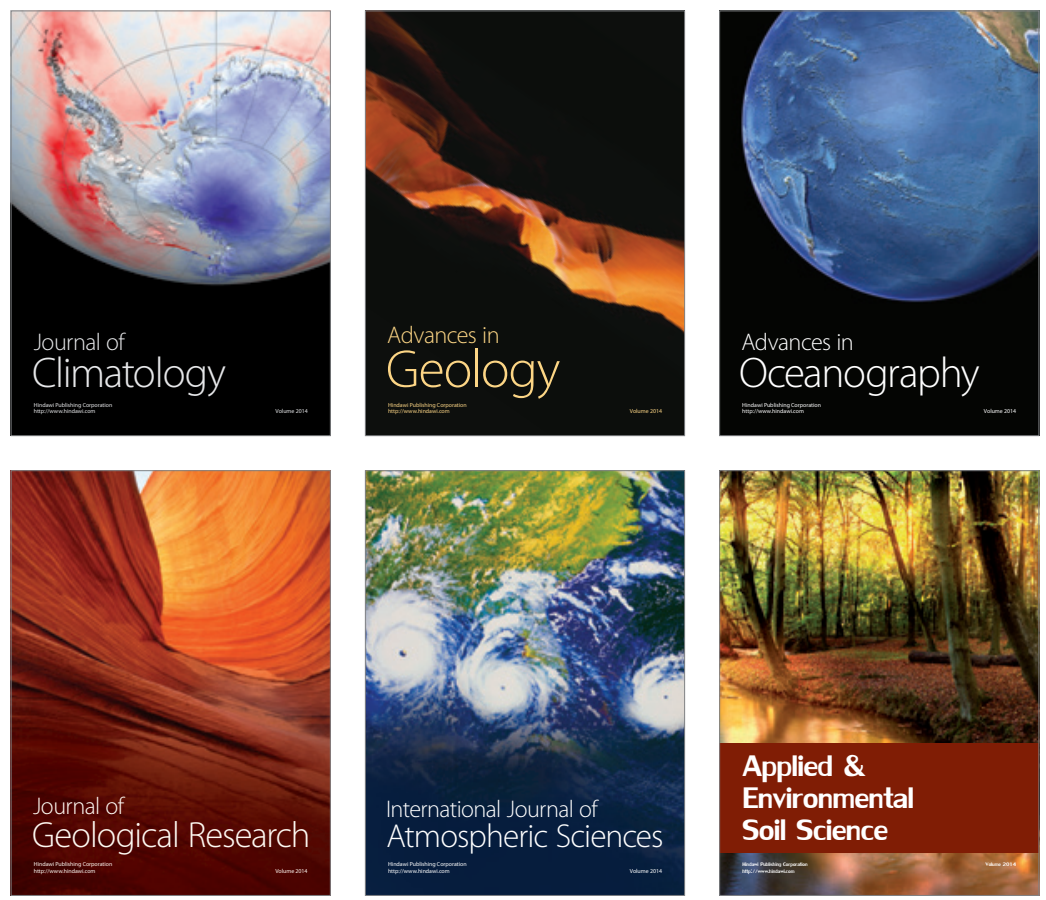
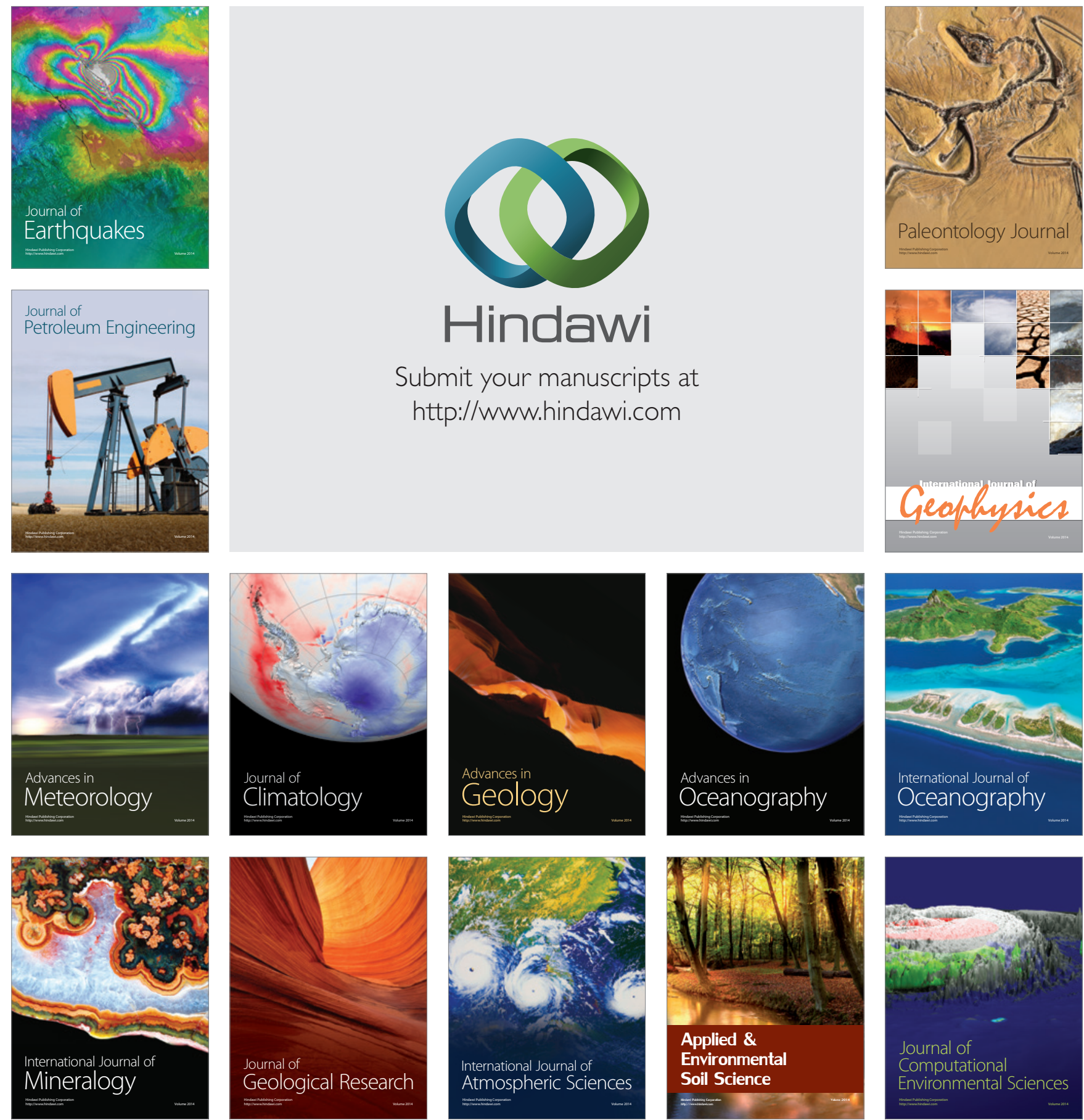